\title{
Indéfini, modalité et généricité dans la Déclaration des Droits de l'Homme
}

Indefinites, Modality and Genericity in the Declaration of Human Rights

\section{Fabienne Martin}

\section{(2) OpenEdition}

\section{Journals}

Édition électronique

URL : http://journals.openedition.org/aad/770

DOI : 10.4000/aad.770

ISSN : $1565-8961$

Éditeur

Université de Tel-Aviv

Référence électronique

Fabienne Martin, «Indéfini, modalité et généricité dans la Déclaration des Droits de l'Homme », Argumentation et Analyse du Discours [En ligne], 4 | 2010, mis en ligne le 15 avril 2010, consulté le 09 octobre 2020. URL : http://journals.openedition.org/aad/770 ; DOI : https://doi.org/10.4000/aad.770

Ce document a été généré automatiquement le 9 octobre 2020.

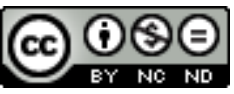

Argumentation \& analyse du discours est mis à disposition selon les termes de la licence Creative Commons Attribution - Pas d'Utilisation Commerciale - Pas de Modification 4.0 International. 


\title{
Indéfini, modalité et généricité dans la Déclaration des Droits de l'Homme
}

\author{
Indefinites, Modality and Genericity in the Declaration of Human Rights
}

\author{
Fabienne Martin
}

\section{Introduction}

1 Comme tout texte de nature juridique, la Déclaration universelle des droits de l'homme (DUDH) est presque exclusivement composée de phrases génériques ${ }^{1}$. Celles-ci peuvent prendre des formes différentes, selon le déterminant utilisé dans les groupes nominaux (le/la, les, un/des, tout, chaque), et la présence ou non d'un verbe modal. De plus en plus de travaux dédiés à la sémantique des phrases génériques montrent que celles-ci sont polysémiques et donc expriment différents types de règles ou de généralisations, et que les différentes formes de phrases génériques contribuent à désambiguïser celles-ci. Curieusement cependant, malgré l'étendue de la littérature consacrée à la généricité et la lente émergence de travaux à l'interface entre sémantique et étude des textes juridiques ${ }^{2}$, les sémanticiens ou les spécialistes du langage juridique ne se sont encore que relativement peu penchés sur la forme des phrases génériques dans les textes légaux, alors que ce type de textes, on va le voir, pose des problèmes intéressants pour toute théorie de la généricité. En outre, parmi les textes légaux, le genre qu'instancie la Déclaration est particulièrement intéressant pour l'étude de la généricité, car le statut des règles qui y sont exprimées est particulièrement difficile à cerner, oscillant encore plus que d'autres types de textes juridiques entre prescription et description.

2 Cet article se divise comme suit. La section 2.1 décrit brièvement les formes préférées ou au contraire évitées des phrases génériques dans la Déclaration. Les sections 2.3 à 4 abordent ces préférences une par une. La section 3 est plus spécifiquement consacrée à l'usage des déterminants, et la section 4 à l'usage de l'indicatif présent « prescriptif ». A chaque fois, on montre en quoi la distribution de ces formes peut être expliquée par les 
théories consacrées aux types de phrases génériques pertinents (celles-ci sont donc brièvement exposées pour les besoins), ou bien au contraire en quoi les théories actuelles ne peuvent rendre compte de toutes les observations linguistiques.

\section{Les phrases génériques dans la Déclaration}

\subsection{Introduction}

3 Une première tendance nette dans les articles de la Déclaration est la préférence pour l'indéfini tout $\mathrm{N}$ ( $\mathrm{N}$ étant mis pour Nom). Ainsi, la forme (1a) est préférée aux formes concurrentes $(1 \mathrm{~b}-\mathrm{d})$ :

(1) a. Tout individu a droit à la vie, à la liberté et à la sûreté de sa personne. (Art. 3)

b. Un individu a droit à la vie, à la liberté et à la sûreté de sa personne.

c. Tous les individus ont droit à la vie, à la liberté et à la sûreté de leur personne.

d. Les individus ont droit à la vie, à la liberté et à la sûreté de leur personne.

4 Ainsi, «tout " indéfini apparaît à plus de vingt reprises ${ }^{3}$. Souvent, lorsqu'une forme différente est préférée, celle-ci apparaît comme un second choix, rendu obligatoire à cause d'un élément bloquant l'utilisation de l'indéfini préféré « tout ». Par exemple, en (2a), l'usage de «tout $\mathrm{N}$ » est bloqué par la relation de comparaison instaurée par « égal », cf. (2b).

(2) a. Tous les êtres humains naissent libres et égaux en dignité et en droit. (Art. 1)

Tous ont droit, sans aucune discrimination, à un salaire égal pour un travail égal.

(Art. 23)

b. ??Tout être humain naît libre et égal en dignité et en droit.

??Tout individu a droit, sans aucune discrimination, à un salaire égal pour un travail égal.

5 Deuxièmement, la forme négative marquée « aucun $\mathrm{N}$ » est souvent préférée à la forme non-marquée « pas de $\mathrm{N}$ » (par exemple, (3a) est préféré à la forme concurrente (3b)):

(3) a. [...] il ne sera fait aucune distinction fondée sur le statut politique, juridique

ou

international du pays ou du territoire dont une personne est ressortissante (Art. 2)

b. Il ne sera pas fait de distinction...

Enfin, les phrases génériques de la Déclaration sont généralement construites sans que la modalité prescriptive soit explicitée par un verbe modal. On a alors affaire à l'indicatif prescriptif, typique du langage juridique. Par exemple, (4a) est préféré à (4b).

(4) a. Tous les enfants [...] jouissent de la même protection sociale. (Art. 25)

b. Tous les enfants doivent jouir de la même protection sociale.

7 Dans les sections 3 et 4 , je vais m'attacher à montrer en quoi ces choix se justifient en général pleinement par, entre autres, le genre qu'instancie la Déclaration et le statut des règles qui s'y voient proclamées. Ainsi, on montrera en quoi les alternatives disponibles auraient moins bien reflété l'esprit du texte (et, le cas échéant, on montrera quelle alternative aurait donné le meilleur second choix). Avant cela, la section 2.2 ouvre quelques réflexions sur les propriétés pragmatiques des phrases génériques d'une part, et des énoncés de droit naturel de l'autre. 


\section{2. Phrases génériques descriptives ou prescriptives et droit naturel}

8 Comme on l'a mentionné dans l'introduction, les phrases génériques diffèrent par le type de généralisations qu'elles expriment, et certaines formes génériques sont plus aptes que d'autres à exprimer certains types de généralisations.

Une opposition classique est opérée entre les phrases génériques descriptives (PGD) qui, typiquement, concluent un raisonnement inductif (bottom up) sur un nombre pertinent de cas particuliers de $\mathrm{P}$ qui sont $\mathrm{Q}$, et les phrases génériques normatives ou prescriptives ( $\mathrm{PGP}$ ), exprimant une règle requérant des $\mathrm{P}$ qu'ils soient $\mathrm{Q}$, et dont la vérité ne dépend d'aucune façon du nombre de $P$ qui sont $Q$. C'est ce deuxième cas qu'illustrent typiquement les énoncés juridiques.

La vérité de la phrase générique n'est pas établie de la même façon dans l'un et l'autre cas. ${ }^{4}$ Très brièvement, lorsqu'elle est descriptive, on procède à l'examen de tout ou partie de l'ensemble P. ${ }^{5}$ Dans le cas d'une phrase générique prescriptive, il faut distinguer deux usages. Sous son usage normatif, une phrase prescriptive crée une norme (et devient vraie par le fait même qu'elle est prononcée), alors que dans son usage assertorique, elle informe l'auditoire à propos du contenu d'un système de normes. Comme le note Hilpinen, cette distinction entre deux usages des phrases normatives remonte au moins à Bentham (1948: 323-24), et recoupe la distinction entre deux types d'énonciation des propositions normatives (Kamp 1979). Ainsi, la proposition normative suivant laquelle les voitures doivent rouler à droite peut être prononcé de manière assertorique, pour informer l'auditoire d'un système de normes de conduite, ou de manière performative, pour donner un ordre et donc créer une norme (et partant, une obligation, cf. Kamp 1979: 263-264, cité par Hilpinen, 1948 : 235).

11 Prima facie, l'usage performatif des énoncés normatifs a quelque chose de paradoxal dans le cas des préceptes de droit naturel, dont les droits de l'homme forment le cas exemplaire. En effet, les propositions juridiques « naturelles » sont censées jouir d'une validité indépendante, supérieure à celle du droit positif ; elles ne peuvent être conférées par un pouvoir politique ; celui-ci ne peut que les reconnaître. La fondation de ces normes censées être immédiatement évidentes se présente comme externe au droit lui-même, comme «naturellement » justifié par l'ordre naturel (Castberg 1970). L'usage descriptif et assertorique semble donc être l'usage qui sied le plus «naturellement» aux énoncés de droit naturel. De fait, l'usage performatif des énoncés normatifs semblerait, par sa conventionnalité, aller à l'encontre du jusnaturalisme des préceptes de droits naturels. C'est aussi l'intuition de Foriers \& Perelman (1973) à propos des droits naturels, pour eux synonymes de droits humains : " Natural rights, being tied by hypothesis to the very nature of man and prior to any social order, cannot be conferred by political authority, but should be recognized and declared by the latter " (mes italiques); "The [natural] rule is then supposed to have existed always, if not in its form at least in its basis, [...] the declarative norm being only the recognition of a preexisting legal principle $» .6$

On verra plus bas qu'en effet, la Déclaration est souvent perçue comme la description de normes qui lui préexistent. D'ailleurs, le verbe «déclarer» lui-même n'est pas performatif. 
Quoiqu'elle n'ait pas elle-même valeur performative, la Déclaration adopte cependant les formes des énoncés prescriptifs qu'elle est censée rapporter. C'est ce qu'on va s'attacher à montrer à travers les sections suivantes.

\subsection{Tout $\mathrm{N}$}

\subsubsection{Un terme de choix libre}

Le déterminant français «tout» est un terme de choix libre (Free Choice Item), tout comme «n'importe quel» ou "quelconque». Intuitivement, en tant que terme de choix libre, la contribution de " tout » à une phrase de type tout $P$ est $Q$ est de spécifier que n'importe quel élément dans la dénotation de $P$ est un candidat approprié pour satisfaire la propriété Q (JT 2004). Ainsi, « tout » spécifie en (1a) que n'importe quel x qui est un individu est appelé à satisfaire la propriété $Q$ « avoir droit à la vie, à la liberté et à la sûreté de sa personne ».

Il est intéressant de noter que dans la version anglaise de la Déclaration, « tout N » n'est pas traduit par le terme de choix libre anyone, mais par le quantificateur universel everyone, plus proche du pronom français «tous». En revanche, la version espagnole est plus proche du texte français sur ce point, puisque «tout $\mathrm{N}$ » $\mathrm{y}$ est généralement traduit par todo N. Je me contenterai, ici, de justifier le choix français, quoiqu'il soit aussi intéressant de motiver en détails la préférence pour everyone en anglais. En deux mots, on peut supposer que l'incongruité de anyone a la même source que celle, en français, de l'autre terme de choix libre "n'importe quel", à savoir une forme de dépréciation de la singularité (Paillard 1997) ${ }^{7}$ :

(5) \#N'importe quel individu a droit à la vie, à la liberté et à la sûreté de sa personne.

\subsubsection{Un indéfini prescriptif}

Un article comme (1a) est constitué d'une phrase générique. Il est facile de le montrer: en règle générale (cf. Kleiber 1977, Tovena \& Jayez 1999, Jayez \& Tovena 2004, Paillard 2000), « tout » fonctionne mal dans une phrase épisodique (non-générique), cf. (6), sauf lorsque $\mathrm{N}$ est modifié par un adjectif ou une relative introduisant une relation causale entre le fait de satisfaire $P$ et le fait de satisfaire $Q$ - en (7), entre le fait d'avoir triché et d'avoir puni.

(6) ??On a renvoyé tout étudiant.

(7) On a renvoyé tout étudiant qui avait triché.

Or « tout » apparaît à plusieurs reprises dans la Déclaration sans modification du type qu'illustre la relative " qui avait triché » en (7), ce qui confirme que les phrases qui le contiennent sont bien génériques.

Il a été noté, pour l'anglais, que les génériques avec noms nus (c'est-à-dire sans déterminant apparent, cf. (8)) sont plutôt interprétées comme des descriptions concluant un raisonnement inductif, alors que les génériques avec indéfinis (cf. (9)) sont investies d'une force normative.

(8) Gentlemen open doors for ladies.

(9) A gentleman opens doors for ladies.

L'observation que Burton (1977) fait à propos de (8)-(9) illustre bien le point : 
if Emile does not as a rule open doors for ladies, his mother could utter (9) and thereby successfully imply that Emile was not, or was not being, a gentleman. Notice that, if she were to utter... (8) she might achieve the same effect (that of getting Emile to open doors for ladies) but would do so by different means... For (8) merely makes a generalisation about gentlemen.

a force normative. Par ailleurs, par son sous-entendu - si un x n'est pas traité comme ayant droit à la vie, à la liberté et à la sûreté de sa personne, ce x n'est pas traité comme un homme - cet énoncé renvoie au topos de l'Untermensch de l'événement historique qui a en partie fondé la Déclaration (cf. Danblon, ce numéro, De Jonge, en prép.). Rappelons-nous le poème en exergue de Si c'est un homme de Primo Levi :

Vous qui vivez en toute quiétude

Bien au chaud dans vos maisons,

Vous qui trouvez le soir en rentrant

La table mise et des visages amis,

Considérez si c'est un homme

Que celui qui peine dans la boue,

Qui ne connait pas de repos,

Qui se bat pour un quignon de pain,

Qui meurt pour un oui ou pour un non.

Étant donné ce contexte historique, où l'humanité s'est vue ébranlée dans ses fondements, la force de l'indéfini, en (1b), tient en outre en ce qu'il sous-entend qu'une société qui ne fait pas en sorte que ses membres aient droit à la vie, à la liberté et à la sûreté de leur personne ne traite pas ses membres comme des hommes, et en ce que cet indéfini fait planer la menace de la comparaison avec l'une des pires sociétés qu'ait connues l'humanité.

La version choisie (1a) est certainement investie d'une force normative comme la variante (1b). Mais la force prescriptive de la variante choisie en « tout » (1a) semble en un sens plus intense, ce que les études sémantiques de « tout " permettent de cerner.

Tout d'abord, Kleiber \& Martin (1977, cités par Tovena \& Jayez 1999) observent que «tout $\mathrm{N}$ » étend la prédication de la phrase à tous les représentants de la phrase, $\mathrm{y}$ compris les moins prototypiques, naturels, fréquents, etc., aux frontières de l'extension de Kadmon \& Landman (1993) ont proposé une analyse similaire pour l'anglais any, par ce qu'ils appellent l'effet « d'élargissement » (widening). On voit évidemment la portée rhétorique d'un tel sous-entendu dans la Déclaration. Ironiquement, C. Muller faisait remarquer, à travers l'expression "Je n'ai pas vu un chat ", que c'était le chat, en français, qui se trouvait aux frontières éloignées de l'ensemble des individus, ce qui 
rend en un sens inutile la Déclaration des Droits des Animaux, dont Danblon (ce numéro) explique par ailleurs l'inefficacité.

Ensuite, Tovena (1996) à propos de any, et à sa suite, Tovena \& Jayez (1999) et Jayez \& Tovena (2004) à propos de «tout », ont montré que les phrases en «tout » n'ont pas d'interprétation descriptive - alors que celle en « un N » en ont au moins une sous leur lecture épisodique ${ }^{9}$. En effet, ces déterminants ne sont pas acceptables lorsque la vérité de la phrase dans laquelle ils apparaissent dépend de l'identité des individus qui forment l'extension de N. Il ne faut pas faire référence à des individus particuliers; les individus doivent pouvoir être différents selon les situations et les mondes. "C'est la possibilité de cette variation qui légitime l'emploi de tout » (Tovena \& Jayez 1999).

Selon leur définition, une phrase est descriptive lorsqu'elle réfère à une situation réelle - comme dans leur exemple (6a) -, ou bien si elle simule une telle référence dans un monde imaginaire, cf. (10) :

(10) Dans cette légende, le chevalier a tué tous les/*tout dragon. (Jayez \& Tovena 2004, leur ex. 10)

Il est évident, dès lors, que "tout " correspond mieux à l'esprit de la Déclaration, censée s'appliquer non seulement au monde actuel, mais aussi à ce qu'il aurait pu être s'il avait été composé d'autres individus, et à ses différentes continuations possibles. L'ensemble des individus auxquels elle s'applique n'est pas connu à l'avance, comme l'exige « tout».

\subsection{Aucun $\mathrm{N}$}

Les études sémantiques synchroniques sur « aucun » sont nettement moins riches que dans le cas de « tout » (voir cependant Heldner 1994). Je me bornerai à montrer, ici, que ce pronom ou ce déterminant renvoie polyphoniquement, à cause de ses propriétés sémantiques, au monde d'avant la Déclaration, plus que ne le ferait son concurrent "pas de ", et que c'est cet effet polyphonique, entre autres, qui justifie son choix. Ce faisant, il renforce l'effet rhétorique initié par l'utilisation du connecteur temporel «désormais » dans le Préambule, qui cimente la frontière temporelle entre l'avant et l'après de la Déclaration (cf. infra et De Jonge, en prép.).

Considérons les contrastes suivants :

(11) a. Je ne connais aucun poème de Paul-Jean Toulet.

b. ??Je ne connais aucun extra-terrestre.

c. Nous n'avons aucun subside.

d. ??Je n'ai aucun crick dans ma voiture.

31 Les phrases contenant un syntagme en " aucun $\mathrm{N}$ » sont partitives en ce sens qu'elles implicitent un ensemble de $\mathrm{N}$ contenant plusieurs éléments (réels ou virtuels) et dont il est envisagé pour chacun qu'il satisfait le prédicat verbal. Par ailleurs, cet ensemble est supposé partagé par les interlocuteurs. Ces contraintes sont immédiatement satisfaites en (11a) ou (11c) : il est facile de partager un ensemble de poèmes de Toulet que j'aurais pu connaître, ou un ensemble de subsides que nous aurions pu obtenir. En revanche, (11b) pose problème parce qu'il requiert un contexte où locuteur et auditoire partageraient une série d'extra-terrestres que je pourrais connaître; en connaître un suffirait déjà à susciter l'étonnement. Notons qu'adressé à un enfant, un énoncé comme «Je t'assure, il n'y aucun extra-terrestre sous ton lit » est plausible dans un contexte partagé où il a été question de plusieurs extra-terrestres différents. De même, (11d) est 
bizarre parce qu'il oblige à se représenter une série de cricks et à imaginer que j'aurais pu les avoir dans ma voiture ${ }^{10}$.

Un peu à la manière d'une série de " ni », la négation de " Aucun N » est en ce sens plus "martelante» parce qu'elle invite à nier la prédication pour plusieurs entités différentes, qu'il oblige à se représenter. Ainsi, (3a) se distingue de (3b) en ce qu'il incite à parcourir la série de distinctions possibles que locuteur et auditoire pourraient avoir communément à l'esprit, et, à nier la prédication pour chacune de celles-ci.

(3) a. [...] il ne sera fait aucune distinction fondée sur le statut politique, juridique ou international du pays ou du territoire dont une personne est ressortissante (Art.

2)

b. Il ne sera pas fait de distinction...

Evidemment, cette inférence invite à se remémorer les distinctions qui ont pu être faites dans le passé, et ainsi à opposer plus concrètement, comme "désormais ", le monde d'avant la Déclaration, et celui qu'elle propose d'atteindre.

"Désormais", on l'a dit, participe à fonder l'opposition entre un monde ancien et le monde nouveau que la Déclaration est censée fonder. De fait, "désormais, P " présuppose que $\mathrm{P}$ entame une "nouvelle ère » au sens strict du terme; c'est pour cela que (13) est curieux (là où (14) ne pose aucun problème).

(13) a. \#Désormais, j'ai pris mon petit déjeuner.

b. Désormais, j'ai passé mon bac.

Un troisième élément qui converge vers la même interprétation « fondationnelle » de la Déclaration est l'utilisation du futur dans plusieurs articles. Fait intéressant, presque toutes les occurrences du futur de la DUDH (repris en a. ci-dessous) ont disparu de la Charte des droits fondamentaux de l'Union européenne ( $\mathrm{CDF}$, repris en b.) s'inspirant des premiers :

(15) a. Nul ne sera tenu en esclavage ni en servitude (DUDH).

b. Nul ne peut être tenu en esclavage ni en servitude (CDF).

(16) a. Nul ne sera soumis à la torture, ni à des peines ou traitements cruels, inhumains ou dégradants (Art. 5, DUDH).

b. Nul ne peut être soumis à la torture, ni à des peines ou traitements inhumains ou dégradants (Art. 4, CDF).

(17) a. Nul ne sera l'objet d'immixtions arbitraires dans sa vie privée, sa famille, son domicile ou sa correspondance [...] (Art. 12, DDH).

b. Toute personne a droit au respect de sa vie privée et familiale, de son domicile et de ses communications (Art. 7, CDF).

De fait, le futur se justifiait en 1948, au commencement de la nouvelle ère de l'aprèsguerre, mais aurait été incongru en 2000, la Charte des droits fondamentaux de l'Union n'ayant pas la vocation fondationnelle de la Déclaration.

\section{L'indicatif prescriptif}

\subsection{Entre prescription et description}

On l'a vu, l'usage généralisé de «tout » fait pencher le texte vers l'interprétation prescriptive. Il n'en reste pas moins, cependant, que malgré la valeur prescriptive des énoncés génériques composant la majorité des articles de la Déclaration, l'intuition du lecteur est que ce texte se présente bel et bien comme la description d'un monde, certes utopique comme l'observe Danblon (ce numéro), mais décrit tout de même. C'est 
d'ailleurs parce qu'il est interprété comme en partie descriptif qu'il est attaqué par les détracteurs des droits de l'homme (ibid., section 3.3) : sous une lecture purement non modale, la Déclaration est « fausse ».

Comme on va le montrer plus bas, il est clair que l'usage de l'indicatif prescriptif est en partie responsable de cet « effet de descriptivité ».

L'indicatif prescriptif (aussi connu sous le nom d'indicatif normatif, cf. Sarcevic 2000) est régulièrement utilisé par les rédacteurs de textes de nature juridique pour exprimer un énoncé déontique. L'expression d'une commande, permission, règle, etc. sans verbe modal explicite prend alors l'aspect de l'expression d'une situation réelle. Comparons à nouveau (4a) et (4b) :

(4) a. Tous les enfants [...] jouissent de la même protection sociale. (Art. 25)

b. Tous les enfants doivent jouir de la même protection sociale.

Les valeurs de l'indicatif prescriptif que l'on trouve par exemple en (4a) ont été explicitement étudiées en philosophie du droit (cf. Villey 1974) ou en linguistique (Ray 1926, Cornu 1990, Gerbe 2006, Deschamps \& Smessaert 2010). D'après les statistiques de ces derniers, les verbes non-modaux à l'indicatif présent constituent près de deux tiers des constructions verbales des Constitutions belge et néerlandaise. Comme le remarquent ces auteurs, une telle fréquence de l'indicatif présent peut paraître étrange, vu que l'on attend des lois qu'elles établissent ce que les citoyens peuvent ou doivent faire, et non ce qu'ils font en réalité.

Suivant Cornu (cité par Deschamps \& Smessaert), l'usage de l'indicatif présent promeut la réception de la loi, et, en outre, est plus diplomatique en déguisant l'ordre sous la prescription :

L'indicatif présent occulte celui qui donne l'ordre et ne brandit pas le pouvoir d'ordonner. C'est une façon plus discrète, plus douce et plus diplomatique de commander. La référence à ce qui est pourrait même faire imaginer que la règle énoncée n'est pas arbitrairement imposée mais naturellement fondée, que le droit est proche de la nature des choses. [...] Dans la même voie, la référence à ce qui se passe montre le sujet de droit agissant, dans le respect de la loi, conformément à la règle, transformant en somme le droit en fait, traduisant la règle dans la réalité [...]. L'indicatif présent anticipe le respect. En cela favoriserait-il la réception du droit. Son emploi est peut-être, en cela, intentionnel.

Autrement dit, suivant l'intuition de Cornu, exprimer la norme par une phrase à l'indicatif (entendue comme non explicitement modalisée) crée un «effet de réalité " qui favoriserait le respect de la règle elle-même. En présentant la règle « en action », le juriste anticipe le respect de celle-ci. Cet effet de réalité de l'indicatif présent est à rapprocher de l'effet rhétorique de l'ekphrasis :

La loi s'efforce aussi d'exciter l'imagination [...] On voit le locataire garnir la maison [...] Les branches des arbres avancent sur la propriété d'autrui (art. 673), les époux vivent séparés (art. 237). Nous y sommes. Ce réalisme est déjà un effet de style (Cornu 1990, cité par Gerbe 2006).

3 De fait, l'effet du «nous y sommes » est exactement ce que vise l'ekphrasis, la figure par excellence de la «vision» (cf., entre autres, Lévy \& Pernot 1997), qui cherche à remporter l'adhésion en touchant par la représentation des choses, et non pas à convaincre en emportant l'intellect.

Pour Ray 1926 également, le choix de l'indicatif se justifie par sa valeur représentative :

ordonner la vie, cela peut se faire non seulement en prescrivant telles activités,

mais en élaborant telles représentations des faits en construisant telle image - 
partiellement représentative, partiellement idéale - des relations que l'on veut régler. Voilà pourquoi la loi s'exprime très souvent sous la forme énonciative ou descriptive, et non pas prescriptive (Ray 1926, cité par Gerbe 2006).

On retrouve l'intuition d'un effet de réalité ailleurs. Suivant Heim (1982) par exemple (citée sur ce point par Greenberg 2003), les phrases génériques prescriptives non explicitement modalisées (cf. 18b) sous-entendent que la règle est suivie dans les faits, à la différence des phrases à verbe modal explicite (cf. 18a).

(18) a. Every dog owner must pay tax on it.

b. A dog owner pays tax on it.

Plus concrètement, selon Heim, seul (18b) suggère que les possesseurs de chien paient effectivement l'impôt sur leur chien. Comme le rappelle Greenberg. Krifka et al. (1995) ont déjà montré qu'il ne peut s'agir d'un entailment, puisqu'il existe des phrases génériques non-modalisées qui ont une lecture purement déontique. Mais il n'empêche qu'elles déclenchent tout au moins l'inférence que la règle est instanciée. Il est intéressant d'observer que l'usage de l'indicatif prescriptif présent est contraint par cet effet de réalité même. Imaginons par exemple une municipalité pour qui offrir une place en crèche à chaque enfant est un idéal à atteindre. On peut imaginer que les auteurs d'une recommandation écrivent (19a), mais (19b) poserait problème dans le même contexte.

(19) a. Tous les enfants doivent bénéficier d'une place en crèche.

b. Tous les enfants bénéficient d'une place en crèche.

En revanche, (19b) serait acceptable si elle avait force de loi appelée à être réellement suivie dans les faits.

C'est sans doute à cause de ce même effet de réalité que la variante (20b) de l'article (20a) serait ressentie comme trop violemment contredite par la réalité. Celle-ci montre en outre qu'il est trop simple de voir entre l'alternance du présent et du futur une simple question stylistique, comme le suggèrent certains manuels de légistique ${ }^{11}$.

(20) a. Nul ne sera soumis à la torture, ni à des peines ou traitements cruels, inhumains ou dégradants.

b. \#Nul n' est soumis à la torture, ni à des peines ou traitements cruels, inhumains ou dégradants.

Nous reviendrons plus loin sur cette implication factuelle de l'indicatif prescriptif. Notons tout de suite que la bizarrerie du présent en (20b) montre déjà que les lois ne s'interprètent pas seulement comme la description atemporelle d'un monde idéal ; en effet, si cela était le cas, le monde réel ne devrait pas faire intrusion dans l'interprétation de la description de ce monde idéal comme c'est le cas en (20b).

Deschamps \& Smessaert (2010) observent une ambiguïté corollaire de l'indicatif prescriptif au plan pragmatique. Tantôt une phrase à l'indicatif prescriptif est simplement comprise comme l'attribution d'un droit, d'une compétence à une entité :

(21) La naturalisation est accordée par le pouvoir législatif fédéral.

51 Ainsi, (21) se borne à attribuer une compétence à une entité, qui est ainsi investie d'un pouvoir discrétionnaire (dont elle use à sa discrétion).

Tantôt le même type de phrase est comprise non seulement comme l'attribution d'un droit ou d'une compétence à une entité $\mathrm{x}$, mais aussi comme l'expression d'une commande (à $\mathrm{x}$ ou à une autre entité $\mathrm{y}$, qui doit ainsi s'assurer que $\mathrm{x}$ bénéficie réellement de ce qui est attribué) :

(22) Le statut légal des fonctionnaires est régulé par un acte du Parlement. 
Ainsi, en (22), la Constitution délègue une compétence au Parlement, mais lui attribue aussi le devoir de produire une règle ou l'autre sur le sujet. Il n'y a alors plus droit discrétionnaire, mais compétence liée («mandatory competence »).

4 La même ambiguïté pragmatique se retrouve dans la Déclaration modulo une petite différence. Contrairement à (21) et (22), ses articles comprennent en majorité un prédicat d'état (jouir de la même protection sociale, être égal devant la loi, etc). En jargon juridique, les normes qui y sont spécifiées sont du type sein-sollen (elles concernent des états de fait), alors que celles qu'on trouve en (21) et (22) sont du type tun-sollen (elles concernent des actes et activités, cf. van Kralingen 1996). D'après von Wright (1983), toute norme de type sein-sollen peut être réécrite en norme de type tunsollen. Ainsi, une norme prescrivant un certain état de fait implique, entre autres choses, que si l'état de fait n'a pas déjà lieu et ne prendra vraisemblablement pas place spontanément, un sujet doit agir de manière à créer cet état de fait ${ }^{12}$. Donc un article sein-sollen de la Déclaration peut s'entendre ou bien comme la simple description d'un droit de $\mathrm{x}$, ou bien en outre comme un ordre d'accomplir l'action assurant ce droit à $\mathrm{x}$.

L'ambiguïté pragmatique que l'on vient de résumer (description simple d'un droit ou combinée à l'attribution d'un ordre) est liée à l'ambiguïté sémantique précédente. En effet, si l'article s'interprète comme l'attribution d'un ordre, alors l'implication factuelle est plus forte que s'il ne s'interprète que comme la description d'un droit. Mais l'interprétation directive n'est pas une cause suffisante pour l'implication factuelle, puisqu'un ordre n'est pas nécessairement suivi d'effets. Nous reviendrons dans la section 3.3 sur l'origine de l'implication factuelle de l'indicatif prescriptif.

\subsection{Les intuitions linguistiques des juristes}

Il est intéressant d'observer que souvent, les juristes eux-mêmes n'arrivent pas à s'accorder sur le sens des énoncés à l'indicatif présent prescriptif, ce qui montre combien l'ambiguïté est difficile à résoudre en contexte. Ainsi, Deschamps \& Smessaert (2010) rapportent qu'à plusieurs reprises, les cours canadiennes ont dû décider si le verbe modal anglais may était chargé d'exprimer un pouvoir discrétionnaire ou une compétence liée (cf. Sarcevic 2000).

En France, l'indicatif de l'article 29 de la Constitution a soulevé une véritable controverse :

(23) Le Parlement est réuni en session extraordinaire à la demande du Premier Ministre ou de la majorité des membres composant l'Assemblée Nationale, sur un ordre du jour déterminé (extrait de l'art. 29 de la Constitution française).

Sans surprise et conformément à son style présidentiel, le Général de Gaulle en son temps interpréta cet article comme l'attribution d'un pouvoir discrétionnaire (Parodi 1982, cité par Savonitto 2008), se dispensant ainsi d'une obligation d'agir. Certains commentateurs ont d'ailleurs, dans son sillon, argué de ce qu'« un indicatif présent n'est pas un impératif » (Parodi 1982: 50), pour appuyer l'idée que l'article n'impose aucune obligation de réunir le Parlement en session extraordinaire: « Le Parlement est réuni » ne signifie pas que le Parlement doit être réuni.

Cependant, d'autres ont considéré que l'emploi de l'indicatif présent appuie justement l'interprétation inverse, suivant laquelle l'article 29 confère au Président l'obligation de convoquer le Parlement en session extraordinaire. Celle-ci résulte notamment de l'emploi de l'indicatif présent. Ceux-ci, citant ironiquement de Gaulle à propos d'un 
autre article de la Constitution, rappelle qu' « en langage juridique, l'indicatif présent a une valeur impérative " (Mollet 1973, cité par Savonitto 2008). Le Code de la route est alors cité en exemple, qui dit « qu'en France la circulation des véhicules se fait à droite. Allez-vous l'interpréter en disant que vous pouvez décider de rouler à gauche si cela vous plaît?» (Mollet 1973, ibid.). L'indicatif «est réuni » est donc utilisé, suivant ces commentateurs, pour attribuer une compétence liée au président. Comme le note Savonitto (2008), cette interprétation plus riche se voit renforcée par une décision du Conseil constitutionnel français à propos d'un autre indicatif présent :

Considérant [...] que l'emploi du présent de l'indicatif ayant valeur impérative, la substitution du présent de l'indicatif à une rédaction formulée en termes d'obligation ne retire pas aux dispositions du nouveau code du travail leur caractère impératif (Conseil constitutionnel, 2007-561, 17 janvier 2008, Journaux officiels, 22.01.2008: 1131'13).

On le voit donc, l'interprétation de l'indicatif prescriptif n'est pas univoque, y compris pour l'auditoire de première main des textes juridiques.

\subsection{L'implication factuelle de l'indicatif prescriptif}

61 La question se pose de savoir pourquoi la règle peut être "présentée en action " par l'indicatif présent dans la charte lorsque le fait est positif (cf. 4a), mais pas lorsqu'il est négatif (cf. 20b) ; les exemples sont repris ci-dessous :

(4) a. Tous les enfants [...] jouissent de la même protection sociale. (Art. 25)

(20) b. \#Nul n'est soumis à la torture, ni à des peines ou traitements cruels, inhumains ou dégradants.

On pourrait supposer que la différence tient à ce que la torture est conçue - à tort ou à raison- comme exceptionnelle dans notre contexte démocratique, alors qu'une protection sociale égale est conçue comme généralisée.

On peut montrer cependant que cela ne peut être à la source du contraste entre (20b) et (4a). Prenons par exemple le cas des violences conjugales; on sait qu'elles sont monnaie courante dans nos pays. Clairement, (24) pose le même problème que (20b) :

(24) a. Nul ne battra sa femme.

b. \#Nul ne bat sa femme.

64 Une autre possibilité est que le futur s'impose pour exprimer des délits futurs par rapport au moment de l'énonciation du droit. Ce serait l'explication de Weinrich 1989 (cité par Gerbe 2006), qui traite du futur dans le Code pénal, plus fréquent que dans le Code civil :

Les rédacteurs du Code pénal sont en particulier convenus qu'ils rédigent ici des dispositions pénales qui ne sont applicables qu'à des délits futurs, c'est-à-dire des délits commis après la promulgation de ces lois. C'est pourquoi ils ont eu pour principe d'utiliser la dimension prospective qu'ils expriment par le futur pour décrire les mesures policières et juridiques à apporter.

Il est fort possible que Weinrich rende bien compte de la préférence pour le futur dans le Code pénal. Cependant, il n'explique pas pourquoi celui-ci s'impose dans les cas qui nous occupent. En effet, d'une part le présent de (25b) ne me semble pas soulever de problème dans un contexte où l'on sait que certains sont condamnés pour des actes délictueux en réalité. Weinrich explique certes la préférence pour le futur dans le texte final, mais pas la possibilité tout de même d'avoir un présent. D'autre part, le futur 
semble plus acceptable (25c), alors que cette phrase ne traite pas de délit. De fait, (25d) provoque un problème en implicitant, contre les faits, que tous les enfants ont un toit.

Ce n'est pas non plus la différence entre les déterminants « tous les » et «nul» qui est en jeu. De fait, les déterminants sont les mêmes qu'en (20b) ou en (4b).

(25) a. Nul ne sera condamné pour des actions ou omissions qui [...] ne constituent

pas un acte délictueux d'après le droit national ou international (variante de l'art.

11).

b. Nul n'est condamné pour des actions ou omissions qui [...] ne constituent pas un acte délictueux d'après le droit national ou international.

c. Tous les enfants auront un toit.

d. \#Tous les enfants ont un toit.

67 Mon hypothèse est que c'est l'opposition entre prédicats factuels - être soumis à la torture, avoir un toit - et prédicats conventionnels - être condamné pour un acte de tel ou tel type, jouir d'une (même) protection sociale - qui est en cause. Les prédicats conventionnels semblent superficiellement décrire une action ou une situation concrète, mais, en réalité, qualifient celle-ci comme instanciant une certaine norme, règle ou convention. Ils oscillent ainsi entre description du fait et qualification de celuici. Ryle 1949 avait bien vu cet aspect duplice des prédicats conventionnels : lorsqu'on dit de $\mathrm{x}$ qu'il a obéi à $\mathrm{y}$, on semble dire deux choses à la fois, puisque $\mathrm{x}$ a fait quelque chose pour obéir à y (voir aussi, sur l'aspect «duel» des prédicats conventionnels, Kearns 2003, qui les appelle criterion predicates).

A mon sens, dans des articles comme (4a) ou (25b), les prédicats conventionnels sont utilisés pour décrire une règle, et non pas pour décrire la réalité qui instancie cette règle. De là vient qu'ils ne donnent pas lieu aux mêmes effets de réalité que les prédicats factuels avec un quantificateur universel comme «tous les ». Ainsi, sous son interprétation normative, (26a) ne requiert pas pour être vrai que tous les enfants obéissent dans les faits à leurs parents, alors que (26b), qui n'a pas d'interprétation normative vu que regarder la télévision n'est pas conventionnel, serait faux si la prédication n'est pas satisfaite par tous dans les faits.

(26) a. Tous les enfants obéissent à leurs parents (prédicat conventionnel)

b. Tous les enfants regardent la télévision (prédicat factuel)

69 L'énoncé (26a) est ambigu : soit il décrit des situations concrètes instanciant une certaine norme, auquel cas il est faux, soit il décrit la norme elle-même, auquel cas il est vrai dans un monde où l'on doit effectivement obéir à ses parents.

70 En conclusion, à cause de son implication factuelle, l'indicatif présent prescriptif pose parfois un problème pragmatique avec les prédicats factuels (plus précisément, lorsque ceux-ci décrivent un événement contraire aux faits du monde actuel), mais pas avec les prédicats conventionnels, car ceux-ci, sous une de leurs interprétations, décrivent une règle, et non la réalité qui instancie cette règle.

\section{Conclusions}

71 On l'a vu, plusieurs choix linguistiques à l'œuvre dans la Déclaration universelle des Droits de l'Homme reflètent l'oscillation caractéristique du texte juridique entre description et prescription. Gerbe (2006) propose de dépasser la contradiction en admettant, dans la lignée de Villey (1974) ou de Danblon (ce numéro), que les énoncés juridiques comme ceux qui composent la Déclaration décrivent le monde idéal à viser. 
Le choix du présent de l'indicatif dans ce type de texte vient ainsi confirmer l'intuition que «la loi n'est autre chose qu'une narration de type particulier» (Sparer \& Schwab 1980).

Il est peut-être aussi opportun de rattacher les ambiguïtés de la Déclaration à son statut juridique. Celle-ci, rappelons-le, n'a pas de véritable portée juridique; elle ne fait que déclarer des droits ${ }^{14}$ et exprimer le désir que ceux-ci constituent un idéal à atteindre. Son ambiguïté modale caractéristique était donc peut-être voulue par ses auteurs. Comme le note Vogel (2009) après Solon (1993), la sous-spécification et l'ambiguïté jouent parfois un rôle sain dans la rédaction d'un texte, surtout dans les contextes diplomatiques : un texte ambigu dans sa construction permet à chaque partie de penser que ses propres intérêts sont protégés.

\section{BIBLIOGRAPHIE}

Aqvist, Lennart. 2003. "Some Remarks on Performatives in the Law », Artificial Intelligence and Law $11: 2-3,105-124$

Batselé, Didier. 2001. Initiation à la rédaction des textes législatifs, réglementaires et administratifs (Bruxelles : Bruylant)

Burton-Roberts, Noel. 1977. « Generic Sentences and Analycity », Studies in Language 1, 155-196

Castberg, Frede. 1970. La philosophie du droit (Paris: Pédone)

Cornu, Gérard. 1990. Linguistique juridique (Paris : Montchrestien)

De Jonge, Emmanuel (en prép.). Les droits de l'homme en discours. Topique et rhétorique des matrices de la modernité (Thèse de doctorat, Université libre de Bruxelles)

Deschamps, Karen (en prép.). De lexicalisatie van rechtsnormen. Deontische modaliteit en negatie in Nederlandstalige regelgevende teksten (Thèse de doctorat, Universiteit Leuven)

Deschamps, Karen \& Hans Smessaert. 2010. « (Non)-Modal Uses of the Present Indicative in Dutch Legislation », De Mulder, Walter, Jesse Mortelmans \& Tanja Mortelmans (éds). Cahiers Chronos (Amsterdam : Rodopi)

Foriers, Paul \& Chaïm Perelman. 1974. « Natural Law and Natural Rights », Wiener, Philip (éd.). Dictionary of the History of Ideas (New-York: Scribner and Sons), 13-14

Gerbe, Rose-Marie. 2006. «Le présent de l'indicatif dans le discours juridique français », Wagner, Anne \& Sophie Cacciaguidi-Fahy (éds). Legal Language and the Search for Clarity (Berne : Peter Lang), 265-302

Greenberg, Yael. 2003. Manifestations of Genericity (New-York : Routledge)

Grewendorf, Guenther \& Monika Rathert. 2009. Formal Linguistics and Law. New Applications of Formal Linguistics (Berlin : Mouton de Gruyter)

Heim, Irene. 1982. «File Change Semantics and the Familiarity Theory of Definiteness ", Rainer Bäuerle, Christoph Schwarze \& Arnim von Stechow (éds). Meaning, Use and the Interpretation of 
Language (Berlin \& New York: de Gruyter) [réimprimé parPortner, Paul \& Barbara Partee (éds). 2002. Formal Semantics. The Essential Readings, 223-248]

Heldner, Christina. 1994. « Le Rôle de la sémantique dans l'interprétation de aucun », Linx numéro spécial, 255-278

Hilpinen, Risto. 2006. « Norms, Normative Utterances and Normative Propositions », Análisis Filosófico $26: 2,229-276$

Jayez, Jacques \& Lucia Tovena. 2004. "Tout as a Genuine Free Choice Item », Corblin, Francis \& Henriëtte de Swart (éds). Handbook of French Semantics (Stanford : CSLI Publications)

Kadmon, Nirit \& Fred Landman.1993. « Any », Linguistics and Philosophy, 353-422

Kamp, Hans. 1979. « Semantics versus Pragmatics », Guenther, F. \& S. J. Schmidt (éds). Formal Semantics and Pragmatics for Natural Languages (Dordrecht: Reidel), 255-287

Kearns, Kate. 2003. « Durative Achievements and Individual-Level Predicates on Events », Linguistics and Philosophy $26: 5,595-636$

Kleiber, Georges \& Robert Martin. 1977. « La quantification universelle en français », Semantikos $2: 1,19-36$

Krifka, Manfred, Francis Pelletier, Gregory Carlson, Alice ter Meulen, Gennaro Chierchia \& Godehard Link. 1995. « Genericity : An Introduction », Carlson, Gregory \& Francis Jeffry Pelletier (éds). The Generic Book (Chicago : University of Chicago Press)

Lévy, Carlos \& Laurent Pernot. 1997. Dire l'évidence. Philosophie et rhétorique antiques (Paris: L'Harmattan)

Mari, Alda \& Fabienne Martin. 2009. « Generic (in)definites and types of judgments », conférence presentée au colloque Genericity : interpretations and uses, Pairs, ENS, mai 2009

Mollet, Guy 1973. Quinze ans après: La constitution de 1958 (Paris : Albin Michel)

Mootz, Francis. 2009. "Perelman's Theory of Argumentation and Natural Law », texte en ligne http://ssrn.com/abstract=1266139

Paillard, Denis. 1997. « N’importe qui, n'importe quoi, n'importe quel N », Langue française 116, 110-114

Paillard, Denis. 2000, « Tout N en français versus vsjakij en russe », Blanco, Xavier (éd.). Détermination et Formalisation, Linguisticae Investigationes Supplementa (Amsterdam : Benjamin), 273-290

Parodi, Jean-Luc. 1982. Les rapports entre le législatif et l'exécutif sous la Cinquième République. 1958-1962 (Paris : Colin)

Ray, Jean. 1926. Essai sur la structure logique du Code civil français (Paris : Alcan)

Ryle, Gilbert. 1990 [1949]. The Concept of Mind (Harmondsworth : Penguin/Peregrine Books) Sarcevic, Susan. 2000. New Approach to Legal Translation (La Haye : Kluwer)

Savonitto, Florian. 2008. « Le pouvoir constituant pose-t-il des normes ? L'exemple des articles 29 et 30 de la Constitution » (Communication au VIIe Congrès français de Droit constitutionnel, septembre 2008), 25-27

Solon, Lawrence. 1993. The Language of Judges (Chicago : University of Chicago Press)

Sparer, Michel \& Michel Schwab. 1980. Rédaction des lois : rendez-vous du droit et de la culture (Québec : Conseil de la langue française) 
Tottie, Gunnel. 1994. « Any as an Indefinite Determiner in Non-assertive Clauses: Evidence from Present-Day and Early Modern English », Kastovsky, Dieter (éd.). Studies in Early Modern English (Berlin : De Gruyter), 413-427

Tovena, Lucia. 1996. Studies on Polarity Sensitivity (Thèse de doctorat, Université d'Edimbourg) Tovena, Lucia \& Jacques Jayez. 1999. « Déterminants et irréférence. L'exemple de tout », Moeschler, Jacques \& Marie-Josée Béguelin (éds). Référence temporelle et nominale (Berne : Lang), 235-268

Van Kralingen, Robert. 1996. Frame-based Conceptual Models of Statute Law (Leiden : Rijksuniversiteit Leiden)

Villey, Michel. 1974. « De l'indicatif dans le droit », Archives de philosophie du droit 19 : 33-61

Vlachou, Evangelia. 2007. Free Choice in and out of Context : Semantics and Distribution of French, Greek and English Free Choice Items (Thèse de doctorat, Universités d'Utrecht et de Paris IV Sorbonne)

Vogel, Carl. 2009. «Law Matters, Syntax Matters and Semantics Matters », Grewendorf, Guenther \& Monika Rathert (éds.) Formal Linguistics and Law. New Applications of Formal Linguistics, (Berlin : Mouton de Gruyter), 25-54

von Wright, Georg Henrik, 1983. Practical Reason (Oxford : Blackwell)

Weinrich, Harald. 1989. Grammaire textuelle du français (Paris : Didier)

Zunzunegui, Edurne. 1994. « Temps verbal, actes de langage et textes juridiques : à propos des versions française et espagnole de l'Acte Unique Européen », Revue romane 23 : 1, 33-49

\section{NOTES}

1. Ce travail fait partie du projet 'B5 Polysemy in a Conceptual System' du Centre de Recherche SFB 732 fondée par la Deutsche Forschungsgemeinschaft. Je remercie Emmanuelle Danblon et Judith Yacar pour des discussions stimulantes et mes relecteurs pour leurs remarques et suggestions sur une version antérieure de ce travail.

2. Voir, par exemple, Grewendorf \& Rathert 2009, Deschamps \& Smessaert 2009, Deschamps (en prép.).

3. Pour l'anglais, il a été noté que any est particulièrement fréquent dans les textes légaux (Tottie 1994).

4. Pour une présentation moins simplifiée de la question de la vérité des énoncés légaux, voir p.ex. Aqvist (2003).

5. Les questions de savoir (1) combien d'éléments de $P$ doivent appartenir à l'ensemble $Q$ pour qu'une phrase générique du type "dét $P$ être $Q$ » soit vraie et (2) combien d'éléments de $P$ doivent être examinés pour établir la vérité de la phrase générique ont été beaucoup été discutées en sémantique formelle. Pour un résumé éclairant, voir Cohen (2002).

6. Outre sa valeur de 'constatif', la Déclaration se présente aussi comme un solemnel expressif: elle est l'expression d'un désir de voir les droits naturels dont elle se fait le reflet comme un idéal à atteindre : «Declarations [of natural rights] are [...] an expression of the wish to protect these rights » (Foriers \& Perelman.). Sur le lien entre la rhétorique de Perelman et le concept de droit naturel, voir Mootz (2009).

7. Sur «n'importe quel », voir les travaux cités de Jayez \& Tovena, ainsi que Vlachou 2007.

8. Sur les relations de dépendance causales dans les phrases génériques avec indéfinis, voir Greenberg 2003 et Mari \& Martin 2009. 
9. Ainsi, « Un gentleman ouvre la portes aux dames "peut décrire un événement particulier. Par ailleurs, Greenberg (2003) a fait l'hypothèse qu'il existe, à côté de la lecture «purement déontique » de (7a) que cerne Burton-Roberts, une lecture de l'indéfini générique à mi-chemin entre la lecture prescriptive et la lecture descriptive (voir p.88). Sous cette lecture, (7a) décrit les cas qui suivent la règle. De la même façon, si je dis a dog owner pays tax on it, je peux soit énoncer une loi (c'est la lecture purement prescriptive, déontique), soit décrire les instanciations de cette loi. Ainsi, dit Greenberg, si j'ai un ami qui veut s'acheter un chien et que je sais qu'il est pauvre, je peux lui dire cet énoncé, car je sais que la règle est suivie dans les faits. Dans ces cas, je n'énonce pas la règle, mais je décris ses instanciations. Crucialement, les phrases génériques en "tout " semblent dépourvues de cette troisième lecture, ce qui les rend prescriptives de manière plus univoque.

10. «Aucun » requiert aussi que l'on discrimine minimalement les éléments de l'ensemble N, autrement dit qu'ils ne soient pas tous conçus comme équivalents. Voyez le contraste suivant :

(12) a. Je n'ai fumé aucune cigarette aujourd'hui.

b. ??Je n'ai aucune cigarette.

(12a) est acceptable parce que l'on différencie facilement les cigarettes que j'aurais pu fumer à 17.00 de celles que j'aurais pu fumer à 17.30, etc. En revanche, (12b) est bizarre sauf dans un contexte marqué où l'on aurait à l'esprit la cigarette que j'ai reçue ce matin de Michel, celle que j'ai récupérée du paquet de la veille, etc.

11. Citons par exemple Batselé (2001) : «Le texte législatif ou réglementaire conjugue parfois le verbe au futur simple. On observe que cette manière d'écrire est rarissime et tend à disparaître. » Notons par ailleurs que le droit civil belge est truffé de futurs simples; ce temps ne semble donc pas partout en déclin comme l'auteur semble le suggérer. Sur l'opposition entre présent et futur de l'indicatif dans les textes de loi, voir aussi Gerbe (2006), qui note une différence très intéressante entre Code civil et pénal français, le second privilégiant l'étude du futur par rapport au premier, ainsi que Zunzunegui (1994) pour une comparaison entre l'usage du futur dans les textes législatifs en français et en espagnol.

12. Je dois ce point ainsi que les références à van Kralingen 1996 et von Wright 1983, à Deschamps 2009.

13. Voir aussi cet extrait du Guide de Légistique du gouvernement français, cité par Gerbe (2006) : «Le présent a valeur impérative. [...] Il n'y a pas lieu, sous prétexte de renforcer le caractère impératif d'une obligation, de recourir au mot "doit"; le simple présent du verbe principal suffit. »

14. C'est d'ailleurs pour permettre le contrôle de son respect effectif qu'a été adoptée la Convention de Sauvegarde des Droits de l'Homme et des Libertés fondamentales.

\section{RÉSUMÉS}

Comme beaucoup de textes de nature juridique, la Déclaration universelle des Droits de l'Homme est composée de phrases génériques oscillant entre les genres descriptif et prescriptif. Cet article étudie les effets argumentatifs des déterminants choisis - en particulier la préférence pour le terme de choix libre «tout » - ainsi que de l'indicatif présent prescriptif, qui investit l'énoncé d'une modalité déontique tout en se présentant comme un énoncé non-modalisé. En particulier, on cherche à expliquer pourquoi, avec certains types de prédicats et pas d'autres, les énoncés à indicatif présent prescriptif déclenchent une implication factuelle. 
Like many texts of juridical nature, the Universal Declaration of Human Rights is composed of generic sentences oscillating between the descriptive and the prescriptive genres. This paper studies the rhetorical effects of the determiners chosen - in particular the preference of the Free Choice Item tout - and the 'prescriptive present indicativ', which endows the sentence with a deontic modality while presenting itself as a non-modalized sentence. In particular, the author explains why the utterances with a prescriptive indicative present trigger an 'actuality entailment' with some predicates and not with others.

\section{INDEX}

Mots-clés : généricité, implication factuelle, indicatif prescriptif, interface sémantique/ rhétorique, item de choix libre

Keywords : actuality entailment, Free Choice item, genericity, prescriptive indicative, rhetorics/ semantics interface

\section{AUTEUR}

\section{FABIENNE MARTIN}

Université de Stuttgart 